\title{
Solid Lipid Nanoparticles of Sulpiride: Improvement of Pharmacokinetic Properties
}

\author{
Mahmoud Mokhtar Ibrahim ${ }^{1,2}$, Abdallah Mohamed Ayoub ${ }^{1}$, Mahmoud Abd Elghany Mahdy ${ }^{1}$, Marwa Helmy Abdallah ${ }^{1,3, *}$ \\ 'Department of Pharmaceutics and Industrial Pharmacy, Faculty of Pharmacy, Zagazig University, Zagazig, EGYPT. \\ 2Department of Pharmaceutics, Oman College of Health Sciences, Pharmacy Program, Ministry of Health, Muscat, OMAN. \\ ${ }^{3}$ Department of Pharmaceutics, College of Pharmacy, Hail University, Hail, KINGDOM OF SAUDI ARABIA.
}

\begin{abstract}
Objectives: In this study, it is hypothesized that lipid carriers could enhance the pharmacokinetic behaviour of Sulpiride (Sul). Different Lipids such as Palmitic Acid (PA), Stearic Acid (SA) and triaplmitin (TA) were used to prepare Solid Lipid Nanoparticles (SLNs) which is then better characterized and tested for its in vivo bioavailability. Methods: SLNs were prepared using film homogenization technique. Different physicochemical parameters such as Homogenization Speed (HS), Homogenization Time (HT) and Sonication Time (ST) were evaluated. Surfactant type and concentration of surfactant, soy lecithin and lipid type were scrutinized. Finally, SLNs pharmacokinetic parameters were evaluated. Results: Sul Entrapment Efficiency (EE) and drug loading were highly associated with solubility in lipid and partition coefficients. The particle size was decreased with an increase in HT, HS and ST. According to lipid type, the EE was high using SA and low using TP. The EE was raised with an increment in the concentration of lipids and soy lecithin. Regarding the in vivo study, Sul SLNs showed 2.64 fold increase in relative bioavailability with higher $C_{\max }(816.22 \mathrm{ng} / \mathrm{ml})$ than the drug suspension
\end{abstract}

form (550 ng/ml). Conclusion: SLNs are considered promising flexible carriers that can be tailored to enhance the solubility, bioavailability and the expected therapeutic response of poorly soluble drugs.

Key words: Nanoparticles, Lipid, Stearic, Sulpiride, Bioavailability, Pharmacokinetics.

\section{Correspondence}

Prof. Marwa Helmy Abdallah,

'Department of Pharmaceutics and Industrial Pharmacy, Faculty of Pharmacy, Zagazig University, Ash Sharqia Governorate-44519, EGYPT.

3Department of Pharmaceutics, College of Pharmacy, Hail University, Hail, KINGDOM OF SAUDI ARABIA.

Phone no: +966 543969452

Email: marwahelmyabdallah@yahoo.com

DOI: 10.5330/ijpi.2019.3.23

\section{INTRODUCTION}

Sulpiride (Sul) "Dogmatil" is a selective antagonist to dopamine D2 receptors. It is one of the benzamide atypical antipsychotic drugs that used in the treatment of psychosis associated with schizophrenia and in major depressive disorders. Moreover, in low doses, it is used in the treatment of anxiety, gastric and duodenal ulcers and mild depression disorders. Sulpiride is rated as class IV in Biopharmaceutical Classification System (BCS). This means that it has poor aqueous solubility and limited intestinal permeability. Previous studies claimed that Sul has a low bioavailability of $27 \%$ after oral administration. ${ }^{1}$ Hence, in order to improve the bioavailability of Sul; different strategies have been investigated, including solid dispersion, microemulsion and nanoparticles. ${ }^{1-3}$

Incorporation of drugs into nanoparticles boosts the bioavailability, prolongs plasma levels and minimizes the variability in the therapeutic outcome. Among nanoparticles, Solid Lipid Nanoparticles (SLNs) were designed to encompass the merits of polymeric particles, liposomes and emulsions while circumventing some of their drawbacks. SLNs are submicron size particles (50-1000 nm), which are composed of solid lipids both at body and room temperature, stabilized by a surfactant at the outer shell. ${ }^{4}$ Recently, Sul was prepared in SLN with different lipids such as Dynasan 118 , Softisan 154 and Imwitor $900 \mathrm{~K}$ by Waheed et al. ${ }^{\circ}$ They used the method of melt homogenization technique for SLN preparation and studied the in vitro release properties and everted sac intestinal permeability, however they didn't study the in vivo effects or pharmacokinetic enhanced properties which is covered in our study.

The aim of this study was to formulate and study different formulation parameters affecting SLNs of Sul as poor soluble drug model using available lipids such as Stearic Acid (SA), Palmetic Acid (PA) and Tripalmetin (TP). The in vivo behavior of the optimum formula was evaluated for the pharmacokinetic parameters using white male rabbits as animal model after oral administration.

\section{MATERIALS}

Brij-78, Tripalmitin (TP) and polyvinyl alcohol (PVA) molecular weight (MW) 13,000-23,000 and partially hydrolyzed (87-89\%) were purchased from Sigma Aldrich (St. Louis, MO, USA). Poloxamer 188 (P188), Compritol 888 ATO (Comp) and Metoclopramide were kindly supplied by EIPICO, 10 $0^{\text {th }}$ of Ramadan City, Egypt. Poloxamer 407 (P407) and glycerylmonostearte (GMS) were kindly supplied by Sigma Pharmaceutical Industries Quesna, Menoufia, Egypt. Sulpiride (Sul) was kindly supplied by Delta Pharm. Co., Egypt. Tween 20 (T20), Tween 80 (T80), Tween 40 (T40), stearic acid (SA), cetyl alcohol (CA) and palmitic acid (PA) were purchased from Adwic, El-Nasr Pharmaceutical Chemicals Co., Egypt. Acetonitrile, ethyl acetate, dichloromethane and methanol (HPLC grade) was purchased from Sigma-Aldrich St. Louis, MO, U.S.A. Sulpiride authentic powder sample was a gift from EPICO.

\section{METHODS}

Film homogenization technique for solid lipid nanoparticles preparation (SLNs)

SLNs were successfully prepared using film homogenization technique. Sulpiride (1\% w/v) and SA (5\%) were homogeneously dissolved in a 
$10 \mathrm{ml}$ of chloroform/methanol mixture (1:1). Then, the organic solvents were completely evaporated under reduced pressure at $60^{\circ} \mathrm{C}$ using a rotary evaporator (Heidolph, Germany) to form a thin lipid film that was kept in a vacuum for $30 \mathrm{~min}$ to remove organic solvent traces. The lipid layer was melted by heating at $80^{\circ} \mathrm{C}$ and hot aqueous phase $\left(80^{\circ} \mathrm{C}\right)$ containing T80 (1\% w/v) sufficient to produce $25 \mathrm{ml}$ of SLNs was added while homogenization at 10,000 rpm using high shearing homogenizer (Ultra Turrax' T 25 basic homogenizer, IKA, Staufen, Germany) for $5 \mathrm{~min}$. The temperature was maintained above the melting point of lipids during the preparation procedure. The produced coarse hot $\mathrm{o} / \mathrm{w}$ emulsion was sonicated for 5 min to obtain Nano emulsion which was cooled to room temperature giving SLNs. ${ }^{6}$ Homogenization speed (HS), homogenization time (HT) and sonication time (ST) were tested for better formulation optimization.

\section{SLNs characterization \\ Effect of different parameters on EE and SLNs size}

T20, T40, T80, P188, P 407, PVA and Brij-78 surfactants were examined. The highest EE and the smallest particle size were the criteria used to select the surfactant of choice for further experiments. The different concentrations of T80 or P188 were examined for their effects on both size and EE. Also, Soy lecithin as a cosurfactant was tested. Finally, the effect of lipid type and Lipid Concentration (LC) on EE and SLNs size was assessed.

\section{Determination of SLNs yield}

The yield was determined gravimetrically after overnight drying of isolated SLNs in a vacuum oven at $30^{\circ} \mathrm{C}$ containing calcium chloride as a desiccant to give an exact weight. The yield was calculated according to the following equation.,

$$
\text { Yield }=\frac{\text { SLNs weight }}{\text { Total initial solid weight }} \times 100
$$

\section{Determination of entrapment efficiency (EE) and drug loading capacity (DL)}

SLNs dispersion was centrifuged at $15,000 \mathrm{rpm}$ for $45 \mathrm{~min}$ and the amount of free Sul in the aqueous phase was estimated by UV analysis after proper dilution with methanol according to the following equations. ${ }^{9,10}$

$$
\begin{gathered}
\mathrm{EE}=\frac{(\mathrm{Wa} \text { disp })}{\mathrm{Wa}} \times 100 \\
\mathrm{DL}=\frac{\text { amount of drug in SLNs }}{(\text { amount of drug in SLNs+lipid })} \times 100
\end{gathered}
$$

\section{Determination of SLN size}

SLN were firstly suspended in deionized water (with tenfold dilution) and analyzed for particle size using light diffraction by Malvern 3000HS (Malvern Instruments Ltd., Worcestershire, UK).

\section{Differential scanning calorimetery}

The Physical Mixture (PM) was prepared by triturating appropriate quantities of Sul and SA using a mortar and pestle. Samples were heated in hermetically sealed aluminum pans over the temperature of $30-300^{\circ} \mathrm{C}$ at a constant rate of $10^{\circ} \mathrm{C} / \mathrm{min}$ under nitrogen purge $(30 \mathrm{ml} / \mathrm{min}){ }^{11}$

\section{In vitro release studies}

The in vitro release of Sul from different SLNs formulations was determined using a modified dialysis membrane diffusion technique. ${ }^{10}$ Briefly, dialysis membrane was soaked in the release media overnight and was stretched over the end of a diffusion glass cell and made water-tight by a rubber band. A measured volume of Sul loaded SLNs (equivalent to $50 \mathrm{mg}$ Sul) was transferred to a glass cylinder. The glass cylinder was then suspended in $250 \mathrm{ml}$ beaker containing $100 \mathrm{ml}$ phosphate buffer ( $\mathrm{pH} 7.4$ ) and maintained at $37 \pm 0.5^{\circ} \mathrm{C}$ under continuous stirring at $100 \mathrm{rpm}$. At different time intervals, $2 \mathrm{ml}$ samples were withdrawn and replaced with pre-warmed sörensen phosphate buffer ( $\mathrm{pH}$ 7.4). The samples were analyzed spectrophotometrically at $\lambda_{\max } 293 \mathrm{~nm}$. The nanoparticles without Sul were treated similarly and used as blank. ${ }^{12}$ Sink conditions were maintained for release studies. ${ }^{13}$

\section{Bioavailability studies of Sulpiride}

White male rabbits $(1.8-2 \mathrm{Kg})$ were acclimatized and kept under constant temperature $\left(25 \pm 2^{\circ} \mathrm{C}\right)$. Animals were treated according to Ethical committee of animal handling in Zagazig University "ECAHZU". Animals were divided into two groups $(n=4)$; the first group was administered Sul suspension while the second group received Sul SLNs (10\% SA, $2.5 \%$ P188, $2 \%$ soy lecithin and $1 \%$ Sul) at a dose of $20 \mathrm{mg} / \mathrm{Kg} .{ }^{1}$ Blood samples were withdrawn at different time intervals from the sinus orbital and centrifuged at $3000 \mathrm{rpm}$ for $10 \mathrm{~min}$ to separate the plasma which was stored at $-20^{\circ} \mathrm{C}$ for analysis.

\section{HPLC analysis for Sul concentration in plasma}

The amount of Sul in each sample was analyzed at room temperature according to Zidan et al. after modification and validation. ${ }^{1}$ The plasma $(1 \mathrm{ml})$ was spiked with $0.1 \mathrm{ml}$ of internal standard (metoclopramide $1.5 \mu \mathrm{g} / \mathrm{ml}$ in mobile phase) and $0.1 \mathrm{ml}$ of a $\mathrm{NaOH}$ solution $(1 \mathrm{~N})$ with vortex mixing for $2 \mathrm{~min}$. Then, mixing with $6 \mathrm{ml}$ of ethyl acetate/ dichloromethane $(5: 1, \mathrm{v} / \mathrm{v})$ and centrifuged at $3000 \mathrm{rpm}$ for $10 \mathrm{~min}$. The supernatant was evaporated under a reduced pressure at $40^{\circ} \mathrm{C}$ until completely dry. The residue was dissolved in a phosphate buffer $(\mathrm{pH} 3)$ and $20 \mu \mathrm{l}$ was automatically injected into the HPLC system. The HPLC system consisted of Agilent 1200 series (Agilent Technologies Inc., Santa Clara, CA, USA) with degasser, autosampler, quaternary pump, Phenomenex $\mathrm{C}_{18} \mathrm{RP}$ column ( $5 \mu \mathrm{m}$ packing, $4.6 \times 150 \mathrm{~mm}$ ), Phenomenex $\mathrm{C}_{18} \mathrm{RP}$ guard column and diode array detector (for multi-wave length and spectral analysis, 8 signals, $20 \mathrm{~Hz}$ data sampling rate). The mobile phase was acetonitrile and $0.01 \mathrm{M}$ phosphate buffer (adjusted to $\mathrm{pH} 3$ using phosphoric acid): Solvent A (90:10 v/v buffer and acetonitrile) and Solvent B (80:20 v/v buffer and acetonitrile). The HPLC run was done at ambient temperature and flow rate of $1 \mathrm{ml} / \mathrm{min}$. The effluent from the column was monitored spectrophotometrically at $212 \mathrm{~nm}$.

\section{Calculation of pharmacokinetics parameters}

The pharmacokinetic parameters were calculated from the plasma drug level data obtained for the individual rabbit per each group and were presented as mean \pm SD. The different pharmacokinetic parameters were calculated using the pharmacokinetic software WinNonlin Standard Edition Version 1.1 (Pharsight, Mountain View, California) using a non- compartmental method.

The relative bioavailability was calculated from the comparison of $\mathrm{AUC}_{0-24}$ of SLNs formulation with that of Sul suspension given orally. ${ }^{6}$

Relative bioavailability $=\frac{\text { AUCOUC at for test }}{\text { AUCOUC for control }} \times 100$

\section{Statistical analysis}

Student's t-test was employed to assess the significance of the difference between the formulations at level $(P<0.05)$ using GraphPad Prism version 5.04 . 


\section{RESULTS}

\section{SLNs Characterization}

The Yields of the obtained SLNs were relatively high in the range of 65\%-95\% (Table 1). Large particles were obtained at HS of 5,000 rpm where the smallest SLNs were obtained at HS of $15000 \mathrm{rpm}$ as shown in Table 1. Also, at HT for $1 \mathrm{~min}$, the EE of Sul was lower than that obtained forth of 3 and $5 \mathrm{~min}$.

Moreover, 10 min HT had resulted in SLNs with lower EE than that obtained at 3 and $5 \mathrm{~min}$.

Table 1 also demonstrated a direct relationship between ST and drug loading up to $10 \mathrm{~min}$ of sonication. After that, further increase in ST up to $15 \mathrm{~min}$ resulted in a decreased drug loading. The optimum formulation conditions for preparation of Sul SLNs were found to be HS of $15,000 \mathrm{rpm}$ for $3 \mathrm{~min}$ followed by sonication for $10 \mathrm{~min}$.

Regarding the surfactant effect on particle size (Table 2), PVA produced the largest particles among different surfactant. ${ }^{12}$

Table 3 demonstrated that increasing surfactant concentration (T80 from 0.5 to $2 \%$ or P188 from $0.5 \%$ to $2.5 \%$ ) resulted in a significant increase in EE.

Table 4 also shows that SLNs stabilized using a mixture of surfactants (soy lecithin, P188 or T80) had a lower particle size in comparison to SLNs having only one surfactant (P188 or T80).

Figure 1 illustrated that greater SLN size and EE were obtained at higher LC. On the other hand, drug loading \% decreased at high LC.

In this study, the lipid type was either in the form of free fatty acid (SA, PA) or in a triglycerides form (TP) and thus the amount of loaded Sul was comparable. At the same lipid concentration, significant differences $(P<0.05)$ in EE and DL between SLNs prepared with different lipid were observed as shown in Figure 1. DSC thermogram of bulk SA showed a sharp melting peak of $70.3^{\circ} \mathrm{C}$, whereas pure Sul exhibited a sharp endothermic peak at $175^{\circ} \mathrm{C}$, which were corresponding to their melting point (Figure 2). There is no significant change in the position of endothermic peaks after running the physical mixture of Sul and lipids. A broad peak with smaller onset temperature suggested that lipids were amorphous in SLNs. However, in the loaded SLNs, the melting peak of Sul was absent.

Table 1: Effect of process variables on physicochemical properties of SLNs (using $5 \%$ stearic acid and $1 \%$ tween 80 ).

\begin{tabular}{ccccccc}
\hline $\begin{array}{c}\text { HS } \\
(\mathrm{rpm})\end{array}$ & $\begin{array}{c}\text { HT } \\
(\mathrm{min})\end{array}$ & $\begin{array}{c}\mathrm{ST} \\
(\mathrm{min})\end{array}$ & EE (\%) & DL (\%) & $\begin{array}{c}\text { Yield } \\
(\%)\end{array}$ & $\begin{array}{c}\text { Particle } \\
\text { size }(\mathrm{nm})\end{array}$ \\
\hline 5,000 & 5 & 5 & $51.00 \pm 4.12$ & $9.25 \pm 0.68$ & 90.30 & 2458 \\
10,000 & & & $45.38 \pm 0.06$ & $8.32 \pm 0.01$ & 88.03 & 1575 \\
15,000 & & & $44.05 \pm 3.99$ & $8.10 \pm 0.67$ & 94.20 & 893 \\
20,000 & & & $37.42 \pm 0.81$ & $6.96 \pm 0.14$ & 91.70 & 956 \\
15,000 & 1 & & $37.73 \pm 2.99$ & $7.01 \pm 0.52$ & 88.80 & 1652 \\
& 3 & & $50.78 \pm 1.78$ & $9.22 \pm 0.30$ & 95.50 & 916 \\
& 10 & & $37.98 \pm 0.65$ & $7.06 \pm 0.12$ & 87.80 & 935 \\
& 3 & 1 & $43.72 \pm 0.49$ & $8.04 \pm 0.08$ & 73.86 & 6058 \\
& & 5 & $50.78 \pm 1.78$ & $9.22 \pm 0.30$ & 95.50 & 916 \\
& & 10 & $52.04 \pm 2.84$ & $9.43 \pm 0.46$ & 85.00 & 513 \\
& & 15 & $40.63 \pm 2.58$ & $7.51 \pm 0.44$ & 77.18 & 689 \\
\hline
\end{tabular}

Table 2: Effect of surfactant type on physicochemical properties of SLNs using $5 \%$ stearic acid.

\begin{tabular}{|c|c|c|c|c|c|c|}
\hline 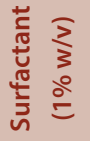 & 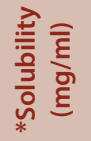 & $\stackrel{\oplus}{\vec{x}}$ & $\frac{\grave{Ð}}{\frac{\mathfrak{d}}{4}}$ & $\frac{\sqrt{a}}{\overrightarrow{0}}$ & 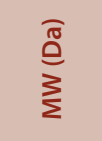 & 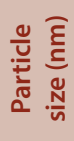 \\
\hline T80 & $\begin{array}{c}1.47 \pm \\
0.30\end{array}$ & 15 & $\begin{array}{c}52.04 \pm \\
2.84\end{array}$ & $\begin{array}{c}9.43 \pm \\
0.46\end{array}$ & 1310 & 513 \\
\hline T40 & $\begin{array}{c}1.38 \pm \\
0.06\end{array}$ & 15.6 & $\begin{array}{c}38.06 \pm \\
2.70\end{array}$ & $\begin{array}{c}7.07 \pm \\
0.46\end{array}$ & 1284 & 593 \\
\hline T20 & $\begin{array}{c}2.20 \pm \\
0.59\end{array}$ & 16.7 & $\begin{array}{c}30.04 \pm \\
2.93\end{array}$ & $\begin{array}{c}5.66 \pm \\
0.52\end{array}$ & 1228 & 496 \\
\hline P188 & $\begin{array}{c}0.94 \pm \\
0.03\end{array}$ & 29 & $\begin{array}{c}58.97 \pm \\
1.77\end{array}$ & $\begin{array}{c}10.55 \pm \\
0.28\end{array}$ & 8400 & 628 \\
\hline P407 & $\begin{array}{c}0.98 \pm \\
0.11\end{array}$ & 22 & $\begin{array}{c}52.21 \pm \\
3.79\end{array}$ & $\begin{array}{c}9.46 \pm \\
0.62\end{array}$ & 12600 & 677 \\
\hline PVA & $\begin{array}{c}2.07 \pm \\
0.03\end{array}$ & 18 & $\begin{array}{c}37.28 \pm \\
3.06\end{array}$ & $\begin{array}{c}6.98 \pm \\
0.53\end{array}$ & $\begin{array}{c}13,000- \\
23,000\end{array}$ & 895 \\
\hline Brij-78 & $\begin{array}{c}1.83 \pm \\
0.12\end{array}$ & 15.3 & $\begin{array}{c}36.80 \pm \\
1.00\end{array}$ & $\begin{array}{c}6.86 \pm \\
0.17\end{array}$ & 1151.54 & 761 \\
\hline
\end{tabular}

*Solubility $(\mathrm{mg} / \mathrm{ml})$ : Solubility of Sulpiride in $1 \%(\mathrm{w} / \mathrm{v})$ aqueous surfactant solution.

Table 3: Effect of surfactant concentration on physicochemical properties of SLNs using $\mathbf{5 \%}$ stearic acid.

\begin{tabular}{ccccc}
\hline $\begin{array}{c}\text { Surfactant } \\
\text { Concentration }\end{array}$ & $\begin{array}{c}\text { Solubility of Sul } \\
(\mathrm{mg} / \mathrm{ml})\end{array}$ & $\mathrm{EE}(\%)$ & $\mathrm{DL}(\%)$ & $\begin{array}{c}\text { Particle } \\
\text { size }(\mathrm{nm})\end{array}$ \\
\hline $0.5 \% \mathrm{~T} 80$ & $0.95 \pm 0.57$ & $38.46 \pm 2.12$ & $8.03 \pm 0.37$ & 1395 \\
$1 \% \mathrm{~T} 80$ & $1.47 \pm 0.3$ & $52.04 \pm 2.84$ & $9.43 \pm 0.46$ & 513 \\
$2 \% \mathrm{~T} 80$ & $2.60 \pm 0.30$ & $57.54 \pm 1.23$ & $10.32 \pm 0.20$ & 471 \\
$3 \% \mathrm{~T} 80$ & $3.01 \pm 0.12$ & $49.75 \pm 2.56$ & $9.05 \pm 0.42$ & 484 \\
$4 \% \mathrm{~T} 80$ & $3.22 \pm 0.09$ & $42.34 \pm 1.29$ & $7.81 \pm 0.22$ & 573 \\
$5 \% \mathrm{~T} 80$ & $3.57 \pm 0.99$ & $32.25 \pm 5.16$ & $6.05 \pm 0.91$ & 528 \\
$0.5 \%$ P188 & $0.77 \pm 0.11$ & $47.43 \pm 2.26$ & $8.67 \pm 0.38$ & 1559 \\
$1 \% \mathrm{P} 188$ & $0.83 \pm 0.03$ & $58.97 \pm 1.77$ & $10.55 \pm 0.28$ & 628 \\
$1.5 \%$ P188 & $0.98 \pm 0.06$ & $62.53 \pm 1.19$ & $11.11 \pm 0.18$ & 585 \\
$2 \% \mathrm{P} 188$ & $1.09 \pm 0.14$ & $64.89 \pm 0.06$ & $11.49 \pm 0.01$ & 511 \\
$2.5 \%$ P188 & $1.35 \pm 0.13$ & $66.96 \pm 0.28$ & $11.81 \pm 0.04$ & 458 \\
$3 \%$ P188 & $1.87 \pm 0.23$ & $60.29 \pm 2.40$ & $10.76 \pm 0.38$ & 584 \\
\hline
\end{tabular}

Soy lecithin addition to SLNs at $2 \%$ concentration resulted in a significant increase in Sul EE from $57.54 \%$ to $70.65 \%$ and from $66.96 \%$ to 76.75 in formulations of T80 and P188 based SLNs, respectively when compared with SLNs formulated without soy lecithin $(P<0.05)$ (Table 4$)$.

Table 4: Effect of soy lecithin concentration on physicochemical properties of SLNs using $\mathbf{5 \%}$ stearic acid.

\begin{tabular}{ccccc}
\hline Surfactant & $\begin{array}{c}\text { Lecithin } \\
\text { (Cosurfactant) }\end{array}$ & EE (\%) & DL (\%) & $\begin{array}{c}\text { Particle } \\
\text { size (nm) }\end{array}$ \\
\hline $2 \%$ T80 & $0 \%$ & $57.54 \pm 1.23$ & $10.32 \pm 0.20$ & 471 \\
2.5 P188 & & $66.96 \pm 0.28$ & $11.81 \pm 0.04$ & 458 \\
$2 \% \mathrm{~T} 80$ & $0.5 \%$ & $60.73 \pm 0.86$ & $10.77 \pm 0.14$ & 439 \\
$2.5 \mathrm{P} 188$ & & $69.43 \pm 0.26$ & $12.19 \pm 0.04$ & 442 \\
$2 \% \mathrm{~T} 80$ & $1 \%$ & $63.75 \pm 0.34$ & $11.31 \pm 0.06$ & 413 \\
$2.5 \mathrm{P} 188$ & & $71.78 \pm 1.09$ & $12.55 \pm 0.17$ & 416 \\
$2 \% \mathrm{~T} 80$ & $1.5 \%$ & $66.97 \pm 1.97$ & $11.81 \pm 0.30$ & 356 \\
$2.5 \mathrm{P} 188$ & & $74.04 \pm 0.52$ & $12.90 \pm 0.08$ & 389 \\
$2 \% \mathrm{~T} 80$ & $2 \%$ & $70.65 \pm 0.70$ & $12.38 \pm 0.11$ & 316 \\
$2.5 \mathrm{P} 188$ & & $76.75 \pm 1.94$ & $13.31 \pm 0.29$ & 348 \\
\hline
\end{tabular}



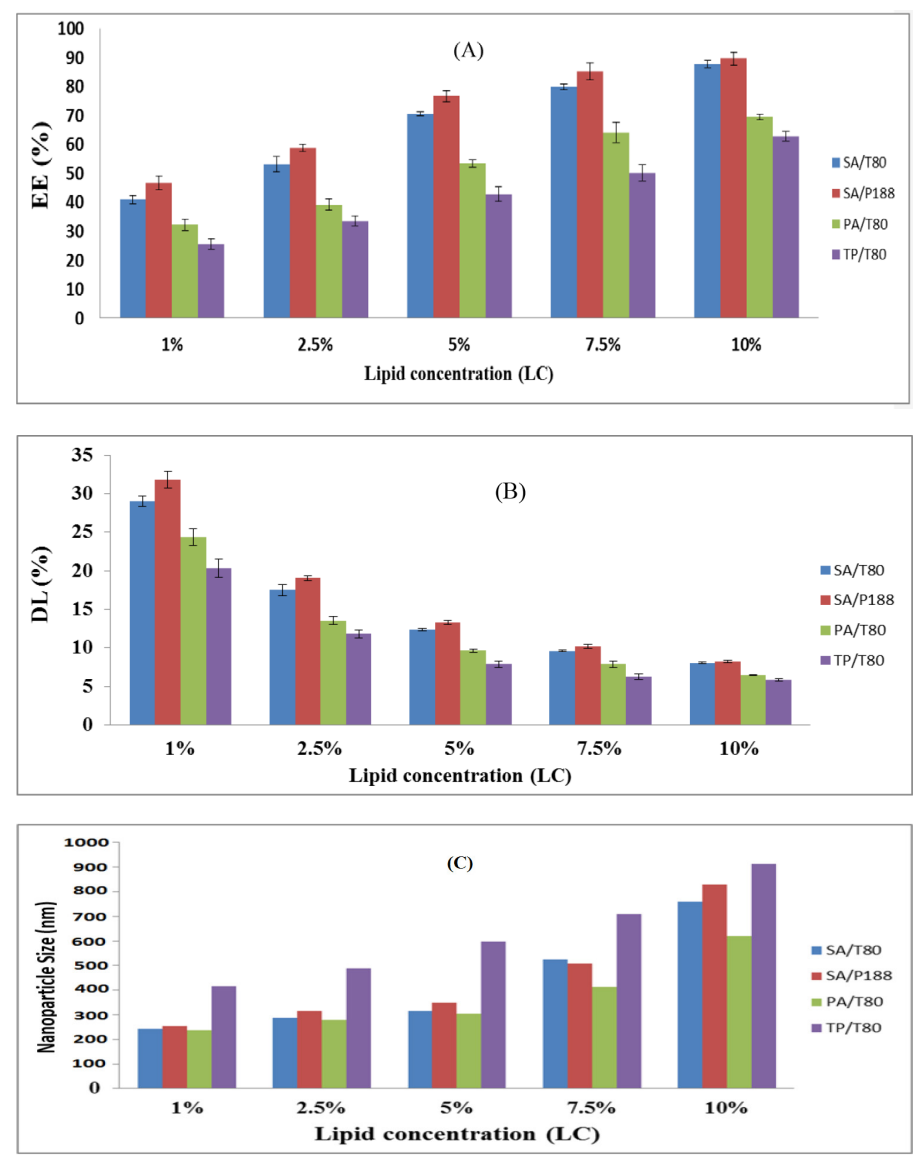

Figure 1: Effect of lipid type and lipid concentration (LC) on EE (A), DL (B) and nanoparticle size (C) of SLNs prepared with different lipid, lipid concentration (LC) and surfactant (S) using $2 \%$ soy lecithin as co-surfactant.

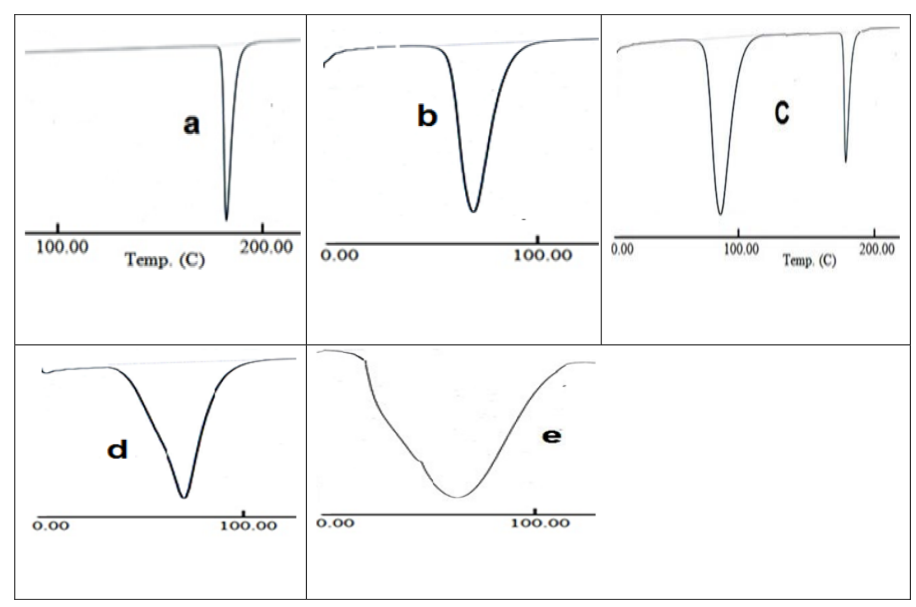

Figure 2: DSC spectra of: a) Sulpiride, b) Stearic acid, c) Stearic acid + Sulpiride PM, d) Blank SLNs, e) Sulpiride SLNs.

\section{In vitro release studies}

Figure 3 depicted that, increasing the LC in the formulation of SLNs from $1 \%$ and up to $10 \%$ showed a significant retarded drug release rates. Sul release was $79 \%, 72 \%, 55 \%, 40 \%$ and $37 \%$ from SLNs of $1 \%, 2.5 \%$, $5 \%, 7.5 \%$ and $10 \%$ LC after $6 \mathrm{~h}$, respectively. Almost $100 \%$ of Sul was released after $12 \mathrm{~h}$ from SLNs of lower LC (1\% and 2.5\%). However, SLNs of greater LC retain more than $20 \%$ of Sul. Those prepared using $10 \%$ LC retain about $40 \%$ of Sul that is gradually released for more $12 \mathrm{~h}$. SLNs of $\mathrm{PA}$ and TP also gave similar results (data not shown).

\section{Oral bioavailability of Sulpiride}

Small particle size and high EE are the decisive criteria which should be in the SLNs tested for in vivo study. The optimum formula for an in vivo testing was selected to have the following composition: $10 \% \mathrm{SA}, 2.5 \%$ P188, 2\% soy lecithin and $1 \%$ Sul.

Figure 4 showed a typical and well-resolved HPLC peaks with retention times of $3.35 \mathrm{~min}$ and 10.23 for Sul and Metoclopramide, respectively. The limit of detection was calculated to be $4.53 \mathrm{ng} / \mathrm{ml}$ while the limit of quantitation was $13.73 \mathrm{ng} / \mathrm{ml}$.

Figure 5 shows the mean plasma concentrations of SLNs formulations versus Sul suspension. Initially, plasma concentration after suspension oral administration was higher than that obtained after SLNs orally administered. Later on, Sul SLNs showed significant higher plasma concentrations than that of the suspension formula. The pharmacokinetics parameters of Sul are summarized in Table 5. The $\mathrm{AUC}_{0-24}$ and $\mathrm{C}_{\max }$ of Sul after oral administration of SLNs formulation were 2.65 fold and 1.48 fold higher than those of Sul suspension, respectively $(p<0.05)$. Statistically, $\mathrm{T}_{\max }$ of SLNs formulation was significantly higher than that

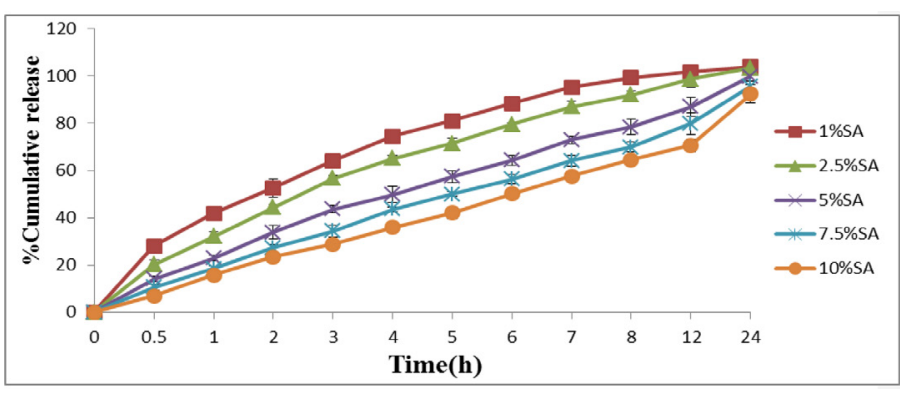

Figure 3: Effect of lipid concentration on the release of Sulpiride from SLNs prepared with $2 \%$ Soya lecithin as co-surfactant.

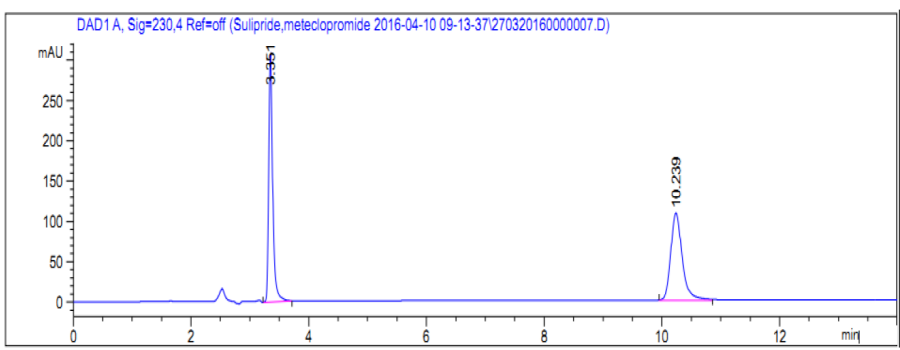

Figure 4: HPLC chromatogram of rabbit plasma containing Sulpiride and Metoclopramide.

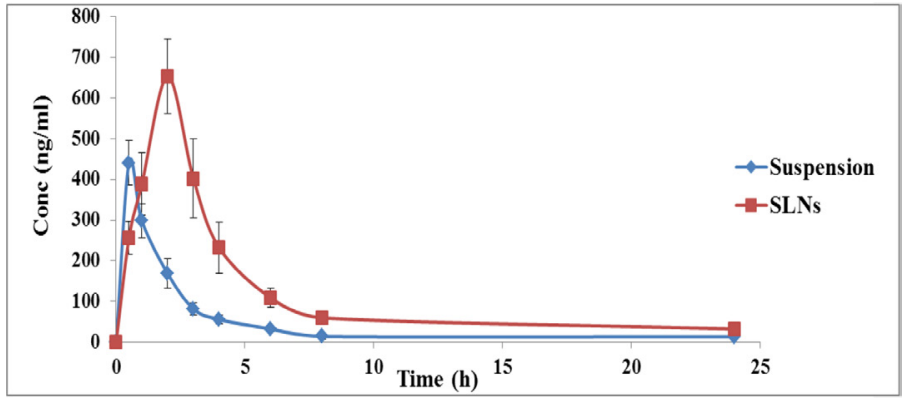

Figure 5: Mean plasma concentration of Sulpiride after oral administration of Sulpiride suspension $(10 \mathrm{mg} / \mathrm{ml})$ and Sulpiride SLNs. 
Table 5: Pharmacokinetic parameters after oral administration of various formulations of Sulpiride $(20 \mathrm{mg} / \mathrm{kg})$ in the rabbit: The values are expressed as mean $\pm \operatorname{SD}(n=4)$.

\begin{tabular}{ccc}
\hline Parameters & Suspension & SLNs \\
\hline $\mathrm{C}_{\max }(\mathrm{ng} / \mathrm{ml})$ & $550.00 \pm 54.44$ & $816.22 \pm 91.43^{*}$ \\
$\mathrm{~T}_{\text {max }}(\mathrm{h})$ & $0.5 \pm 0.00$ & $2.00 \pm 0.00^{*}$ \\
$\mathrm{~K}_{\text {ele }}\left(\mathrm{h}^{-1}\right)$ & $0.13 \pm 0.04$ & $0.09 \pm 0.01^{*}$ \\
$\mathrm{t}_{1 / 2}(\mathrm{~h})$ & $5.16 \pm 1.02$ & $7.72 \pm 0.63^{*}$ \\
$\mathrm{AUC}_{0-24}\left(\mathrm{ng} \cdot \mathrm{h} \cdot \mathrm{ml}^{-1}\right)$ & $1272.88 \pm 90.30$ & $3371.30 \pm 383.78^{*}$ \\
$\mathrm{AUC}_{0-\infty}\left(\mathrm{ng} \cdot \mathrm{h} \cdot \mathrm{ml}^{-1}\right)$ & $1326.65 \pm 115.12$ & $3605.86 \pm 434.65^{*}$ \\
$\mathrm{MRT}(\mathrm{h})$ & $5.17 \pm 0.83$ & $6.90 \pm 0.86^{*}$ \\
$\mathrm{CL}(\mathrm{ml} / \mathrm{min})$ & $252.69 \pm 22.06$ & $93.36 \pm 10.10^{*}$ \\
Relative bioavailability (\%) & --------- & 264.86
\end{tabular}

${ }^{*}$ Significantly different at $P<0.05$

of suspension form indicating the prolonged release pattern of SLNs $(p<0.05)$. The terminal half-life $\left(\mathrm{t}_{1 / 2}\right)$ and the Clearance $(\mathrm{Cl})$ of Sul after administration of Sul suspension was about $5.16 \mathrm{~h}$ and $252.69 \mathrm{ml} / \mathrm{min}$, respectively. Whereas, with SLNs, 1.5 fold increase in $t_{1 / 2}$ and 2.62 fold decrease in $\mathrm{Cl}$ were observed (Table 5).

\section{DISCUSSION}

SLNs were successfully prepared using film homogenization technique with relatively high yield. The slight loss of SLNs might take place at any step during preparation or separation of SLNs such as sticking of the lipid to the glass wall. ${ }^{14}$ The loss of solids was higher in high lipid concentration samples than samples with lower lipid concentration. This may be attributed to a higher propensity of particle aggregation at larger lipid concentration samples. ${ }^{15,16}$ On the other hand, at lower HS, the shearing force was insufficient to comminute the droplet size of an emulsion to nanoemulsion. ${ }^{17}$ However, too high speed may raise the collision between the newly formed particles producing large and irregular shaped particles. ${ }^{16}$ The decrease in EE with longer HT could be due to distortion of the outer stabilizer coat and lipid core leading to leaching of the drug to the external phase ${ }^{16}$ However, homogenization for longer times may destabilize the particles resulting in particles aggregation ${ }^{16}$ Also, higher ST could lead to the disruption of lipid core causing drug molecule escaping to external phase. ${ }^{4}$ The difference in EE using different surfactants might be attributed to the solubilizing potential of the surfactant in the aqueous phase. In addition, the high content of hydroxyl groups in PVA could result in greater intramolecular interaction via formation of hydrogen bonds between or inside molecules and hence, greater aqueous phase viscosity and coalescence of adjacent particles. ${ }^{14}$ Increasing surfactant concentration would minimize drug leaching because of the higher viscosity of the aqueous phase leading to higher EE. ${ }^{18}$ On the other hand, increasing surfactant concentration above $2 \%$ can result in micellar solutions of the drug and lower EE. ${ }^{5}$

Addition of soy lecithin as a co-surfactant could improve the drug solubility and incorporation in the lipid matrix by providing more space for incorporating the drug. ${ }^{19}$ Moreover, the combination of hydrophilic and hydrophobic surfactants in which soy lecithin preferably interspersed between the lipid layers while P188 or T80 pile up at the outer surface of SLNs might hinder any particle agglomeration contributing to a decrease in particle size. ${ }^{20}$

EE was arranged in the descending order ( $\mathrm{SA}>\mathrm{PA}>\mathrm{TP})$. All TP based formulations had relatively low $\mathrm{EE}$ due to the absence of a free carboxylic group. Fatty acids (SA and PA) produced significantly smaller particles than triglycerides at the same lipid concentration. This is reasonable since TP has higher MW than SA and PA. Fatty acid molecules are about one-third the size of triglycerides composed of the same fatty acids. ${ }^{21}$ The fatty acid chain length had a significant effect on the particle size of SLNs. Figure 1 illustrated that SA based nanoparticles had a larger size than PA counterparts. The higher melting point and MW of SA might result in a more viscous dispersed phase that would be less homogenized effectively at the same conditions and in turn larger size. ${ }^{13}$ The Lipid Concentration (LC) is from the factors that should be considered when formulating SLNs for its great effects on drug EE. Figure 1 revealed that, the lowest EE was observed at 1\% LC in all used lipids. The lipid concentration was insufficient to entrap the available drug. On the other hand, the highest EE was obtained at 10\% LC. This might be justified by different reasons. Firstly, the higher LC provided more hydrophobic rooms to incorporate more drug molecules. ${ }^{22}$ Secondly, higher LC was associated with higher viscosity and faster solidification of lipid core. ${ }^{14}$ In contrary, DL significantly decreased with increasing LC. The drug/lipid ratio was decreased with an increase in LC at the same drug concentration. ${ }^{23}$ The homogenization efficiency might decrease with higher viscous dispersion and the distribution of sonication waves was lower; thereby the cavitation force would be inadequate for efficient size reduction. ${ }^{14}$

Furthermore, incorporation of Sul into SLNs could reduce both the onset temperature and melting point of SA. ${ }^{24}$ The Sul DSC peak absence (Figure 2) could be due to the solubility of the drug in SA and drug could be transformed into an amorphous form. Taking into consideration the Sul release from SLNs, the higher partition coefficient of Sul in the lipids might be the main reason for drug sustained release behavior. ${ }^{25}$ The longer carbon chain length in SA might help to retain lipophilic drugs causing slower release rates. ${ }^{13}$ Taking into consideration the results of Sul release, the bioavailability studies showed greater $\mathrm{T}_{0.5}$ and lower drug clearance than suspension form. SLNs increased Sul bioavailability by more than two fold in comparison to suspension form. Sul suspension dissolved in the intestinal tract and absorbed directly into systemic circulation reaching the peak concentration quickly at $0.5 \mathrm{~h}$ while Sul in SLNs slowly diffused through lipid layer into GIT. Therefore, the intact Sul SLNs released the drug gradually into the blood circulation. This was due to the fact that SLNs could enhance the solubility and intestinal permeability of poorly soluble drugs. ${ }^{5}$

\section{CONCLUSION}

In this work, the effects of different parameters on SLNs formulation were probed to obtain SLNs with optimized properties. Three different lipids namely, stearic acid, palmitic acid and triaplamitin were explored as lipid carriers in SLNs preparation. The entrapment efficiency and particle size were highly dependent on the studied parameters. Stearic acid produced the higher entrapment efficiency and drug loading among tested lipids. The prepared formula was efficient carrier for modulating the pharmacokinetic profile of Sul more than two fold increase as much relative bioavailability as suspension form. The prime components in SLNs such as poloxamer 188 and stearic acid together with the nanoscale size may lead to the higher bioavailability and hence negate the need for higher oral doses. Taken together with the ready availability of materials and easy preparation, SLNs are a possible strategy in oral drug delivery.

\section{ACKNOWLEDGEMENT}

The authors would like to thank Dr Mahmoud El Batriq in the Department of Pharmacology, Faculty of Pharmacy, Zagazig University, for his help and advice during the bioavailability study and statistics. 


\section{CONFLICT OF INTEREST}

The authors declare no conflict of interest.

\section{ABBREVIATIONS}

Sul: Sulpiride; SLNs: Solid lipid nanoparticles preparation; EE: Entrapment Efficiency.

\section{REFERENCES}

1. Zidan AS, Emam SE, Shehata TM, Ghazy FE. Pediatric suppositories of sulpiride solid dispersion for treatment of Tourette syndrome: In vitro and in vivo investigations. AAPS Pharm Sci Tech. 2015;16(3):645-55.

2. Ibrahim WM, AIOmrani $A H$, Yassin AE. Novel sulpiride loaded solid lipid nanoparticles with enhanced intestinal permeability. Int J Nanomedicine. 2014;9:129-44.

3. Ayoub AM, Ibrahim MM, Abdallah MH, Mahdy MA. Sulpiridemicroemulsions as antipsychotic nasal drug delivery systems: In-vitro and pharmacodynamic study. J Drug Deliv Sci Technol. 2016;36:10-22.

4. Mehnert W, Mader K. Solid lipid nanoparticle. Production, characterization and applications. Adv Drug Deliv Rev. 2001;47(2-3):165-196.

5. Waheed MI, AIOmrani AH, Eldeen ABY. Novel sulpiride-loaded solid lipid nanoparticles with enhanced intestinal permeability. Int J Nanomedicine. 2014;9:129-44

6. Hosny KM, Banjar ZM, Hariri AH, Hassan AH. Solid lipid nanoparticles loaded with iron to overcome barriers for treatment of iron deficiency anemia. Drug Des Dev Ther. 2015;9:313-20.

7. Parveen R, Ahmad FJ, Iqbal Z, Samim M, Ahmad S. Solid lipid nanoparticles of anticancer drug andrographolide: Formulation, in vitro and in vivo studies. Drug Dev Ind Pharm. 2013;40(9):1206-12.

8. Gardouh AR, Gad S, Ghonaim HM, Ghorab MM. Design and characterization of glycerylmonostearate solid lipid nanoparticles prepared by high shear homogenization. British J Pharmaceutical Res. 2013;3(3):326-46.

9. Tiwari R, Pathak K. Nanostructured lipid carrier versus solid lipid nanoparticles of simvastatin: Comparative analysis of characteristics, pharmacokinetics and tissue uptake. Int J Pharm. 2011;415(1-2):232-43.

10. Omwoyo W, Ogutu B, Oloo F, Swai H, Kalombo L, Melariri P, et al. Preparation, characterization and optimization of primaquine-loaded solid lipid nanoparticles. Int J Nanomedicine. 2014;9:3865-74.

11. Ebrahimi HA, Javadzadeh $Y$, Hamidi M, Jalali MB. Repaglinide-loaded solid lipid nanoparticles: Effect of using different surfactants/stabilizers on physicochemical properties of nanoparticles. Daru. 2015;23(1):46-57.
12. Xie S, Zhu L, Dong Z, Wang X, Wang Y, Li X, et al. Preparation, characterization and pharmacokinetics of enrofloxacin-loaded solid lipid nanoparticles: Influences of fatty acids. Colloids Surf B: Biointerfaces. 2011;83(2):382-7.

13. Jain $A$, Singh SK, Singh $Y$, Singh S. Development of lipid nanoparticles of diacerein, an antiosteoarthritic drug for enhancement in bioavailability and reduction in its side effects. J Biomed Nanotechnol. 2013;9(5):891-900.

14. Singh S, Dobhal AK, Jain A, Pandit JK, Chakraborty S. Formulation and evaluation of solid lipid nanoparticles of a water soluble drug: Zidovudine. Chem Pharm Bull. 2010;58(5):650-5.

15. Kim BD, Na K, Choi HK. Preparation and characterization of solid lipid nanoparticles (SLN) made of cacao butter and curdlan. Eur J Pharm Sci. 2005;24(23):199-205.

16. Shah B, Khunt D, Bhatt $H$, Misra M, Padh H. Application of quality by design approach for intranasal delivery of rivastigmine loaded solid lipid nanopar ticles: Effect on formulation and characterization parameters. Eur J Pharm Sci. 2015;78:54-66.

17. Battani $\mathrm{S}$, Pawar $\mathrm{H}$, Suresh $\mathrm{S}$. Evaluation of oral bioavailability and anticance potential of raloxifene solid lipid nanoparticles. J Nanosci Nanotechnol. 2014;14(8):5638-45

18. Ravi P, Aditya N, Kathuria H, Malekar S, Vats R. Lipid nanoparticles for oral delivery of raloxifene: Optimization, stability, in vivo evaluation and uptake mechanism. Eur J Pharm Biopharm. 2014;87(1):114-24.

19. Aboud M, Elkomy H, Ali A, ElMenshawe F, AbdElbary A. Development, optimization and evaluation of carvedilol loaded solid lipid nanoparticles for intranasal drug delivery. AAPS Pharm Sci Tech. 2016;17(6):1353-65.

20. Tran TH, Ramasamy T, Truong DH, Choi G, Yong S, Kim O. Preparation and characterization of fenofibrate-loaded nanostructured lipid carriers for oral bioavailability enhancement. AAPS Pharm Sci Tech. 2014;15(6):1509-15.

21. Triplett M, Rathman JF. Optimization of $\beta$-carotene loaded solid lipid nanopar ticles preparation using a high shear homogenization technique. J Nanopart Res. 2009;11(3):601-14.

22. Gidwani B, Vyas A. Preparation, characterization and optimization of altretamine-loaded solid lipid nanoparticles using Box-Behnken design and response surface methodology. Artifi Cells Nanomed Biotechnol. 2014;44(2):571-80.

23. Pradhan $M$, Singh $D$, Singh M. Influence of selected variables on fabrication of Triamcinolone acetonide loaded solid lipid nanoparticles for topical treatment of dermal disorders. Artif Cells Nanomed Biotechnol. 2016;44(1):392-400.

24. Chalikwar SS, Belgamwar VS, Talele VR, Surana SJ, Patil MU. Formulation and evaluation of Nimodipine-loaded solid lipid nanoparticles delivered via lymphatic transport system. Colloids Surf B Biointerfaces. 2012;97:109-16.

25. Manjunath K, Venkateswarlu V. Pharmacokinetics, tissue distribution and bioavailability of clozapine solid lipid nanoparticles after intravenous and intradeodenal administration. J Control Release. 2005;107(2):215-28.

Cite this article: Ibrahim MM, Ayoub AM, Mahdy MA, Abdallah MH. Solid Lipid Nanoparticles of Sulpiride: Improvement of Pharmacokinetic Properties. Int. J. Pharm. Investigation. 2019;9(3):122-7. 